\title{
EFECTOS A CORTO PLAZO DE LA CONTAMINACIÓN ATMOSFÉRICA SOBRE LA MORTALIDAD: RESULTADOS DEL PROYECTO EMECAM EN CARTAGENA, 1992-96 *
}

\author{
José Jesús Guillén Pérez (1), Lluís Cirera Suárez (1), Luis García-Marcos Álvarez (2), Enrique \\ Jiménez Torres (3), Xavier Barber Vallès (1), María José Martínez García (4) y Stella Moreno \\ Grau (4).
}

(1) Dirección General de Salud. Consejeria de Sanidad y Politica Social. Murcia.

(2) Unidad de Investigación Clínico-epidemiológica. Cartagena.

(3) Servicio de Medio Ambiente. Ayuntamiento de Cartagena.

(4) Departamento de Ingeniería Química-Cartagena. Universidad de Murcia.

$\left(^{*}\right)$ Este trabajo cuenta con una beca del Fondo de Investigaciones Sanitarias (Expediente núm 97/0051-09).

\section{RESUMEN}

Fundamento: Los problemas de contaminación atmosférica se han venido percibiendo en la ciudad de Cartagena desde la década de los setenta, con episodios puntuales de altos niveles de $\mathrm{SO}_{2}$ y partículas. Nos proponemos evaluar, utilizando la metodología del proyecto $E M E C A M$, los efectos agudos de la contaminación atmosférica por $\mathrm{SO}_{2}$ y partículas sobre la mortalidad diaria en la ciudad de Cartagena de 1992 a 1996.

Métodos: se relacionan las defunciones diarias por todas las causas excepto las externas, en población general y en la de 70 y más años, por causas cardiovasculares y por causas respiratorias, con la contaminación atmosférica por dióxido de azufre y partículas, en el período1992-1996, utilizando modelos de Poisson autorregresiva que controlan por estacionalidad, meteorología, calendario, gripe, eventos especiales y retardos.

Resultados: En el período en estudio se ha producido una disminución de la contaminación por $\mathrm{SO}_{2}$, con respecto a años anteriores, lo que no ha sido evidente para la contaminación por partículas. Los análisis muestran asociaciones significativas en las defunciones totales sin accidentes en mayores de 69 años con el valor promedio de partículas, éstas con las defunciones cardiovasculares del mes de mayo a octubre. En el semestre frío, encontramos asociación estadísticamente significativa positiva en el valor máximo horario diario de las particulas y las muertes, por enfermedades cardiocirculatorias y respiratorias. Sin embargo no hay consistencia en las asociaciones al evaluar la fiabilidad de los modelos.

Palabras clave: Mortalidad. Contaminación atmosférica. Efectos ambientales adversos, Efectos a corto plazo. Series temporales. Regresión de Poisson. Autocorrelación. Enfermedades cardiovasculares. Enfermedades respiratorias. Dióxido de azufre. Partículas en suspensión.

Correspondencia:

José Jesús Guillén Pérez

Servicio de Salud Pública. Cartagena

Dirección General de Salud

Plaza de San Agustín, 3. 30201 - Cartagena

Tel. 968 502573; Fax 968502570

Correo electrónico: josej.guillen@carm.es.
ABSTRACT

\section{The Short-term Impact of Air Pollution on the Mortality. Results of the EMECAM Project in Cartagena, 1992-96}

Background: The problems of air pollution became noticeable in Cartagena in the seventies, high $\mathrm{SO}_{2}$ and particle levels having been reached from time to time. Our aim is to assess, using the EMECAM methodology, the acute impact of $\mathrm{SO}_{2}$ and particle air pollution on the daily death rate of the city of Cartagena in the 1992-1996 period.

Methods: A daily listing is provided of the total number of non-accidental deaths within the population as a whole and for those over age 70 , the cardiovascular and the respiratory deaths due to dioxide and particle air pollution for the 1992-1996 period using autoregressive Poisson models which control seasonality, weather, time of year, flu, special events, and time lags.

Results: In the period under study, there has been a drop in the $\mathrm{SO}_{2}$ air pollution as compared to previous years, which was not as marked for the particles. The analyses reveal significant relationships in the total non-accidental deaths in those over age 69 , with the average particle count and thole particles with cardiovascular deaths for the months of May to October. In the six-month period of the year, when the weather is cold, we found a positive statistically significant relationship to exist in the maximum daily hourly value of the particles and the deaths due to cardiocirculatory and respiratory diseases. However, there is no consistency in the between on assessing the reliability of the models.

Key words: Mortality. Air pollution. Adverse environmental impact. Short-term impact. Time series. Poisson regression. Autocorrelation. Cardiovascular diseases. Respiratory diseases. Sulfur dioxide. Particles in suspension. 


\section{INTRODUCCIÓN}

Los problemas de contaminación atmosférica se han venido percibiendo en la ciudad de Cartagena desde el comienzo de la década de los setenta ${ }^{1}$, con situaciones alarmantes debidas a las emisiones industriales y a las condiciones meteorológicas de dispersión de los contaminantes, que han ocasionado episodios puntuales de altos niveles de $\mathrm{SO}_{2}$ y partículas.

En los años de 1977 a 1980 las medias anuales se situaban entre los 200 y 300 $\mu \mathrm{g} / \mathrm{m}^{3}$ (figura1). Así, el casco urbano de Cartagena y parte de su término municipal fueron declarados por el Real Decreto 1197/1979, como zonas de aplicación de las medidas previstas para áreas de atmósfera contaminada en la ley de Protección del Medio Ambiente Atmosférico. En 1978, en una estación medidora de la contaminación de origen industrial, las medias mensuales fueron superiores a los $600 \mu \mathrm{g} / \mathrm{m}^{3}$ y en 155 días se superaron los $400 \mu \mathrm{g} / \mathrm{m}^{3}$ de media diaria.
Esta situación llevó al Ayuntamiento de la Ciudad a establecer un Servicio de Medio Ambiente para conocer tanto los niveles de inmisión como las emisiones de las industrias, y al establecimiento de medidas para solucionar la contaminación del medio atmosférico. Esto condujo a que, tras un episodio de contaminación por $\mathrm{SO}_{2}$ en diciembre de 1990, se estableciese un Plan Operativo de Intervención sobre la contaminación atmosférica de origen industrial, en la que, tras recibir información en la Central de Datos de Medio Ambiente, se insta a las empresas a disminuir carga o a parar los procesos cuando se observa que la media de 24 horas puede aumentar por encima de los límites establecidos.

No son numerosos los estudios que relacionan la mortalidad y la contaminación en Cartagena, aunque es de destacar el que analiza patrones diferenciales de mortalidad $\mathrm{e}$ incidencia de cáncer en el ámbito de Cartagena ${ }^{2}$ con otras áreas geográficas regionales que se consideraban menos expuestas a

Figura 1

Medias anuales de $\mathrm{SO}_{2}\left(\mu \mathrm{g} / \mathrm{m}^{3} \mathrm{~N}\right)$. Cartagena 1973-1997

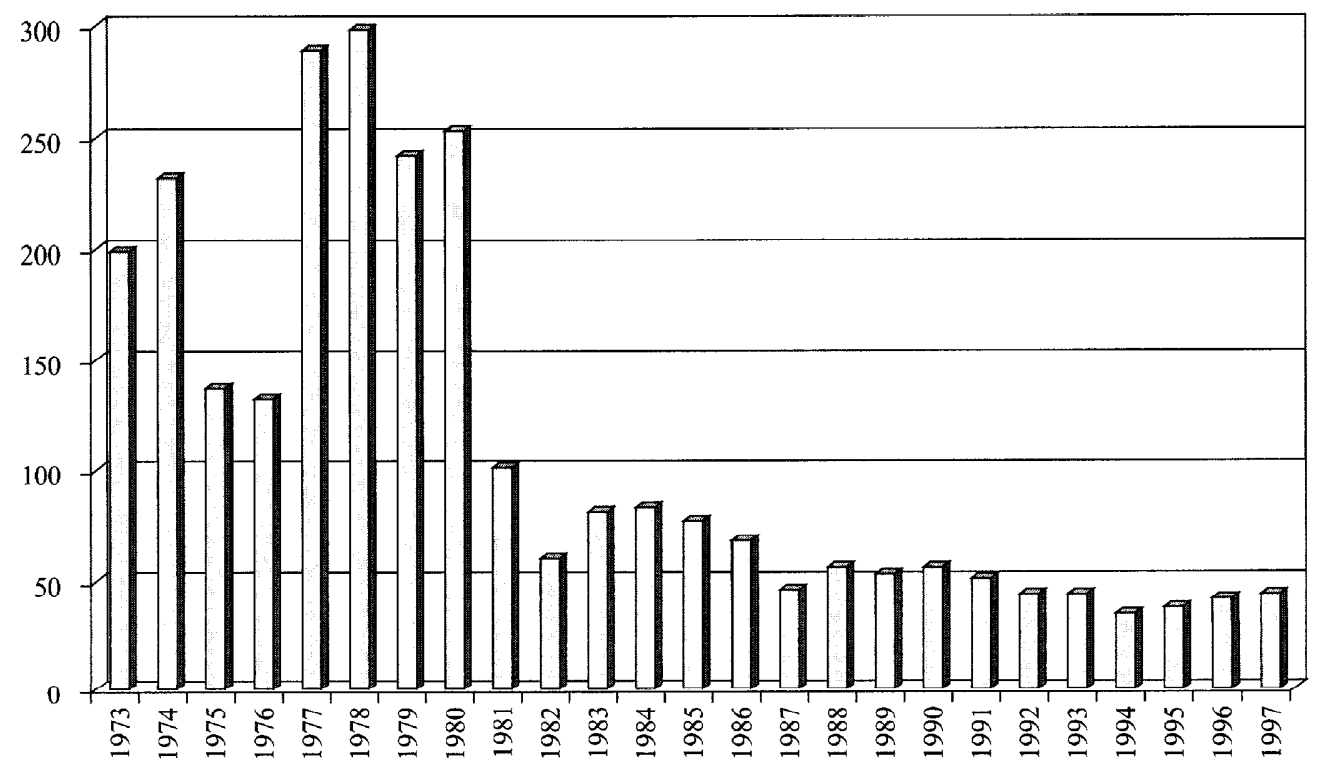


contaminación, observándose una mayor mortalidad por cáncer de pulmón, próstata y vejiga en hombres, y por enfermedad coronaria en ambos sexos en 1984-85, y de incidencia para los mismos cánceres en hombres en 1983-85.

También hay que reseñar el estudio que se hizo entre 1989 y $1991^{3}$, en el que se concluye que había un exceso de afluencia a urgencias de casos de asma cuando el $\mathrm{SO}_{2}$ superaba los $80,6 \mu \mathrm{g} / \mathrm{m}^{3}$.

Nos proponemos evaluar el impacto de la contaminación atmosférica sobre la mortalidad diaria en la ciudad de Cartagena, en el período de 1992 a 1996, analizando la asociación de los efectos agudos por $\mathrm{SO}_{2}$ y partículas sobre distintos tipos de causas de defunción, utilizando la metodología del proyecto $E M E C A M$...

\section{MATERIAL Y MÉTODOS}

El Municipio de Cartagena abarca una superficie de $557 \mathrm{~km}^{2}$ con una población de 180.553 habitantes en el padrón corregido de 1995 . El de 1996 refleja 170.483 habitantes y el de 1991168.023 . No significa que haya habido oscilaciones en el número de habitantes sino que, dado el bajo registro de los mismos, hay que esperar las correcciones para conocer la población de forma más exacta. Se puede decir que ésta ha permanecido estable en cuanto a número, pero con el consiguiente envejecimiento. Los menores de 5 años representan el $4,47 \%$ y los que tienen 65 o más años el $14,1 \%$, siendo el $66,16 \%$ los de 5 a 49 años y el $15,27 \%$ los de 50 a 64 años.

Utilizamos las defunciones diarias de los habitantes residentes ocurridas en el municipio de Cartagena, información cedida por el Centro Regional de Estadística de Murcia. Un pequeño porcentaje ( $4 \%$ en 1995) de los habitantes fallece en la ciudad de Murcia, donde se ubica el hospital regional de referencia (Virgen de la Arrixaca). Estudiadas sus causas de defunción, se decidió excluir- los por presentar patologías no ligadas al posible efecto biológico de la contaminación (sobre todo accidentes de tráfico o de evolución crónica como algún tipo de tumor).

Siguiendo el protocolo del proyecto $E M E C A M^{4,5}$ utilizamos como variables meteorológicas la temperatura media diaria y la humedad relativa diaria (media de la humedad a las $0,7,13$, y 18 horas) facilitadas por el Instituto Nacional de Meteorología de la cstación medidora de Capitanía General de la Zona Marítima del Mediterráneo, ubicada en el casco antiguo de la ciudad.

En el período estudiado, el Centro de Control del Departamento de Medio Ambiente del Ayuntamiento de Cartagena disponía de 12 estaciones medidoras, la mayoría de ellas con funcionamiento automático y con registro informático desde su puesta en marcha en los últimos años. Cinco de ellas, las más antiguas, habían registrado todo el período 1992-1996, pero la instalada en el Puerto de Cartagena se trasladó en 1995. La ubicada en Alumbres (ER2) no está situada en zona urbana, ya que es una pedanía que se encuentra junto al complejo industrial de Escombreras. Las tres restantes, Lo Campano (ER1), Bastarreche (ER3) y Pista deportiva-San Ginés (ER5), cumplían el requisito de estar en zona urbana, por lo que fueron seleccionadas (figura 2 ).

Como variables de contaminación utilizamos el $\mathrm{SO}_{2}$ y las partículas totales en suspensión, expresadas como la media de 24 horas y valor horario máximo diario (max. hor.), medidas por las tres estaciones automáticas de forma instantánea y que cumplían con el requisito de ser urbanas.

Decidimos comenzar en enero de 1992, ya que de septiembre a noviembre de 1991 en las estaciones se sustituyó el analizador de partículas existente (se cambió el nefelómetro SIGRST por un gravimétrico TEOM 
Figura 2

Situación de la red de estaciones medidoras de contaminación en Cartagena ( 1 a 5 )

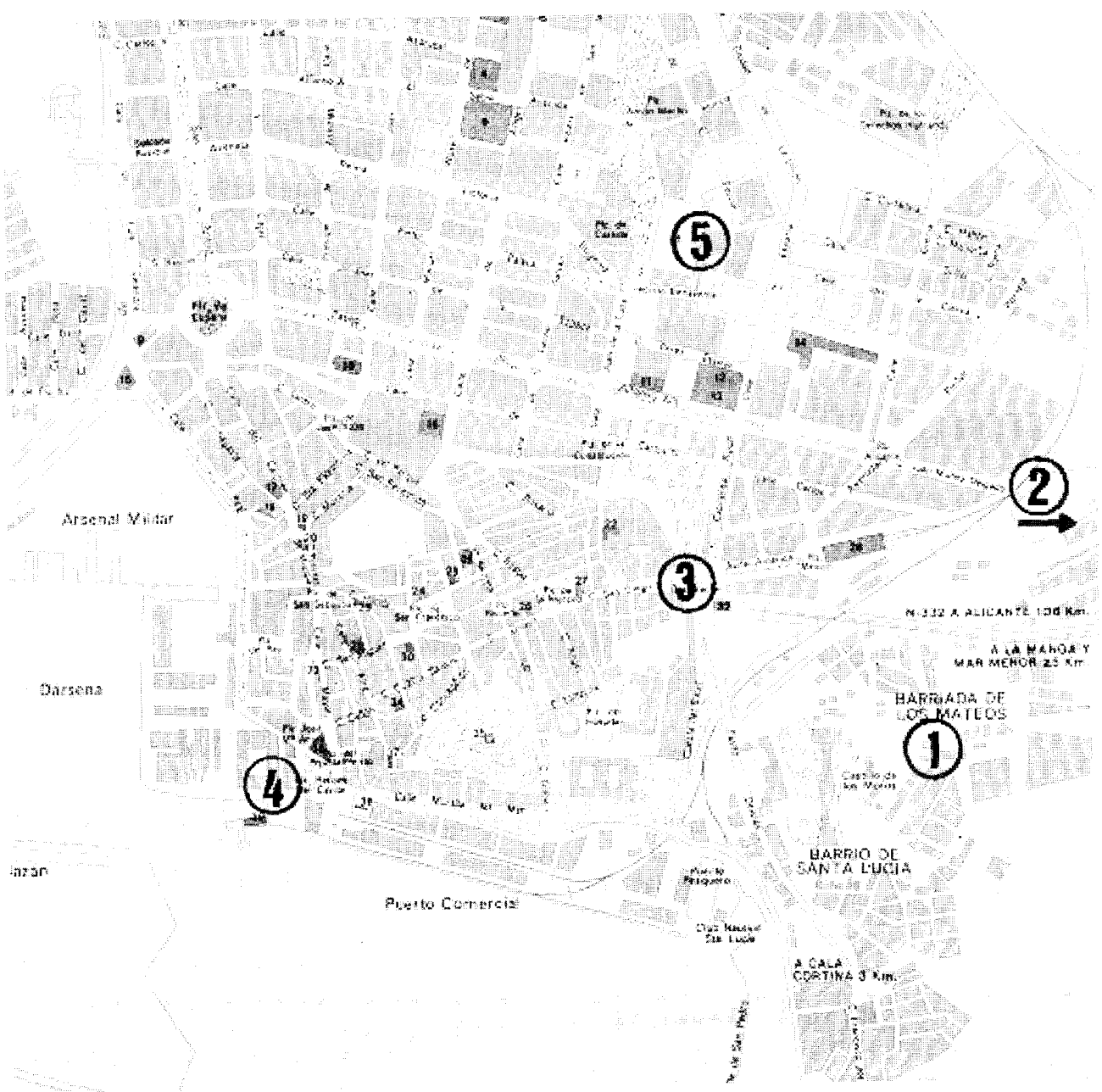

1 Lo Campano

2 Alumbres

3 Bastarreche

4 Puerto

5 San Ginés

1400), habiéndose encontrado diferencias en los resultados con los dos métodos.

Los casos de gripe en la ciudad se obtuvieron del Sistema de Declaración de Enfermedades de Declaración Obligatoria de la Comunidad Autónoma.
Plan Operativo de intervención municipal

La existencia del Plan Operativo ${ }^{6}$, único en su configuración, ha ocasionado que se evite en gran medida la aparición de días con alta contaminación, como ocurría en el pasado. El Plan Operativo compromete a las 
empresas, vistas las cifras de contaminantes en las estaciones, a que procedan a bajar carga (se interviene si el $\mathrm{SO}_{2}$ es de 400 $\mu \mathrm{g} / \mathrm{m}^{3}$ en 15 minutos, $250 \mu \mathrm{g} / \mathrm{m}^{3}$ en 3 horas o $200 \mu \mathrm{g} / \mathrm{m}^{3}$ en cuatro horas o las partículas son de $130 \mu \mathrm{g} / \mathrm{m}^{3}$ durante cuatro horas) 0 parar la producción de la empresa (si la situación no se corregía en una hora con la bajada de carga o si las condiciones ambientales hacían prever que no se solucionara el episodio).

\section{Análisis estadísticos}

El estudio se realizó tomando el día como unidad de análisis. Las correlaciones entre variables meteorológicas y contaminantes, las efectuamos mediante el coeficiente de correlación de Spearman ${ }^{7}$. La asociación entre los niveles de contaminantes y el número de fallecimientos se probó mediante modelos de Poisson autorregresiva para cada tipo de mortalidad, en el que se incluyen la variedad de contaminante atmosférico, la estacionalidad, la meteorología, el calendario, la incidencia de gripe, los eventos especiales, y sus respectivos retardos. El posible efecto del contaminante sobre las defunciones se explora hasta el quinto retardo. La estimación puntual del riesgo (RR) y su intervalo de confianza al $95 \%$, están medidas en número de defunciones diarias por cada $10 \mu \mathrm{g} / \mathrm{m}^{3}$ de contaminante ${ }^{8}$. En los semestres cálidos y fríos elaboramos dos modelos: a) con el mismo modelo de todo el período más la interacción contaminante/semestre; b) modelos específicos, distintos al modelo del período anual total, aplicando de nuevo el protocolo para los datos del semestre.

Para comprobar la consistencia de la parametrización de los modelos se realizó el análisis de fiabilidad, que consiste en reproducir el modelo obtenido introduciendo, en primer lugar, el tipo de contaminante atmosférico y, secuencialmente, las variables indicadas en el protocolo, tanto para los modelos de todo el período como para los semes- tres, comprobando las variables que son distintas, y las magnitudes y significación de los coeficientes de regresión para el contaminante.

\section{RESULTADOS}

En el periodo de estudio Cartagena ha presentado unas condiciones climáticas acordes a su situación mediterránea, con un valor mínimo de la temperatura media diaria de $7,2^{\circ} \mathrm{C}$ y máxima de $30,8^{\circ} \mathrm{C}$. La humedad relativa en el período fue del $75,24 \%$, habiendo disminuido desde 1995 en los que se manifestó una pertinaz sequía.

Ha sido evidente la disminución de $\mathrm{SO}_{2}$ a lo largo del período (tabla 1); así la media anual de $\mathrm{SO}_{2}$ de las tres estaciones medidoras utilizadas en 1992 fue de $51,69 \mu \mathrm{g} / \mathrm{m}^{3} \mathrm{y}$ en $1997 \mathrm{de} 23,5 \mu \mathrm{g} / \mathrm{m}^{3}$. También ha existido una tendencia decreciente de la media de partículas aunque no de forma tan llamativa $\left(65,93 \mu \mathrm{g} / \mathrm{m}^{3}\right.$ en 1992 y $49,77 \mu \mathrm{g} / \mathrm{m}^{3}$ en 1997), sin embargo, los valores máximos diarios siguen presentando niveles similares a lo largo del período.

Es necesario investigar la influencia del Plan Operativo en el comportamiento de los contaminantes. El número de días con intervención en el Plan Operativo en el período fue de $352(19,27 \%)$ presentando una distribución estacional debido a su relación con las condiciones climáticas (figura 3 ). Los días de intervención presentaron de forma significativa valores más altos de los contaminantes medidos que los días que no hubo intervención $\left(\mathrm{SO}_{2} 59,8 \mu \mathrm{g} / \mathrm{m}^{3}\right.$ frente a $40 \mu \mathrm{g} / \mathrm{m}^{3}$, partículas $73,1 \mu \mathrm{g} / \mathrm{m}^{3}$ frente a $51,7 \mu \mathrm{g} / \mathrm{m}^{3}, \mathrm{SO}_{2}$ max.hor. $185,22 \mu \mathrm{g} / \mathrm{m}^{3}$ frente a $95,96 \mu \mathrm{g} / \mathrm{m}^{3}$, partículas max.hor. $253 \mu \mathrm{g} / \mathrm{m}^{3}$ frente a $\left.160,39 \mu \mathrm{g} / \mathrm{m}^{3}\right)$. La media de defunciones sin accidentes en el período ha sido de 3,5 diarias, oscilando de 4 en invierno a 3,2 en verano, siendo la media diaria de las defunciones por causas respiratorias de 0,4 ; las cardiovasculares de 1,4 y de 2,4 en las personas de 70 y más 
Tabla 1

Valures medios anuales de $\mathrm{SO}_{2}$ y Partículas $\left(\mu \mathrm{g} / \mathrm{m}^{3} \mathrm{~N}\right)$ según la estación captadora. Cartagena 1985-1997

\begin{tabular}{|c|c|c|c|c|c|c|c|c|c|c|c|c|}
\hline \multirow[b]{2}{*}{ Estaciones captadores } & \multicolumn{6}{|c|}{$\mathrm{SO}_{2}$} & \multicolumn{6}{|c|}{ Particulas } \\
\hline & ERI & $E R 3$ & ER5 & $E R 2$ & ER4 & $\begin{array}{l}\text { Media } \\
\text { ER1, 3, } \\
5\end{array}$ & $E R I$ & ER3 & ER5 & $E R 2$ & $E R 4$ & $\begin{array}{c}\text { MEDIA } \\
E R I, 3 \\
5\end{array}$ \\
\hline 1985 & 119 & 75 & - & 38 & - & 97 & - & - & - & - & - & - \\
\hline 1986 & 107 & 48 & - & 48 & - & 77,5 & - & - & - & - & - & 一 \\
\hline 1987 & 98 & 32 & 36 & 36 & 30 & 55,33 & - & - & - & -- & & \\
\hline 1988 & 124 & 62 & 31 & 23 & 41 & 72,33 & 58,5 & 78,9 & 43,05 & 76,89 & 30,18 & 60,15 \\
\hline 1989 & 99 & 99 & 40 & 48 & 34 & 79,33 & 59,78 & 71,17 & 55,74 & 50,78 & 62,71 & 62,23 \\
\hline 1990 & 82 & 101 & 22 & 45 & 29 & 68,33 & 56,79 & 68,95 & 59,57 & 61,23 & 70,98 & 61,77 \\
\hline 1991 & 76 & 87 & 27 & 29 & 36 & 63,33 & 36,54 & 71,67 & 69,44 & 63,12 & 75,41 & 59,22 \\
\hline 1992 & 46,85 & 80,38 & 27,85 & 35,81 & 29,64 & 51,69 & 56,79 & 99,23 & 41,77 & 43,95 & 58,30 & 65,93 \\
\hline 1993 & 34,21 & 83,0 & 30,26 & 40,17 & 34,92 & 49,16 & 44,56 & 80,02 & 19,32 & 42,41 & 58,55 & 47,97 \\
\hline 1994 & 28,38 & 60,07 & 26,27 & 40,18 & 20,43 & 38,24 & 51,97 & 99,08 & 42,17 & 52,67 & 65,52 & 64,41 \\
\hline 1995 & 30,04 & 55,78 & 19,01 & 49,46 & - & 34,94 & 50,78 & 91,82 & 28,82 & 48,08 & - & 57,14 \\
\hline 1996 & 44,3 & 67,19 & 23,14 & 34,08 & - & 44,88 & 48,08 & 56,8 & 30,56 & 42,12 & - & 45,15 \\
\hline 1997 & 26,1 & 30,2 & 14,2 & 13,6 & - & 23,5 & 49,5 & 64 & 35,8 & 45,3 & - & 49,77 \\
\hline Media del periodo & 70,38 & 67,74 & 26,98 & 36,95 & 31,87 & 58,12 & 51,39 & 78,16 & 42,62 & 52,66 & 60,24 & 57,39 \\
\hline
\end{tabular}

ER1 Lo Campano.

ER2 Alumbres.

ER3 Bastarreche.

ER4 Puerto.

ER5 San Ginés.

Fïgura 3

Media mensual de intervenciones municipales operativas en Cartagena 1992-1996

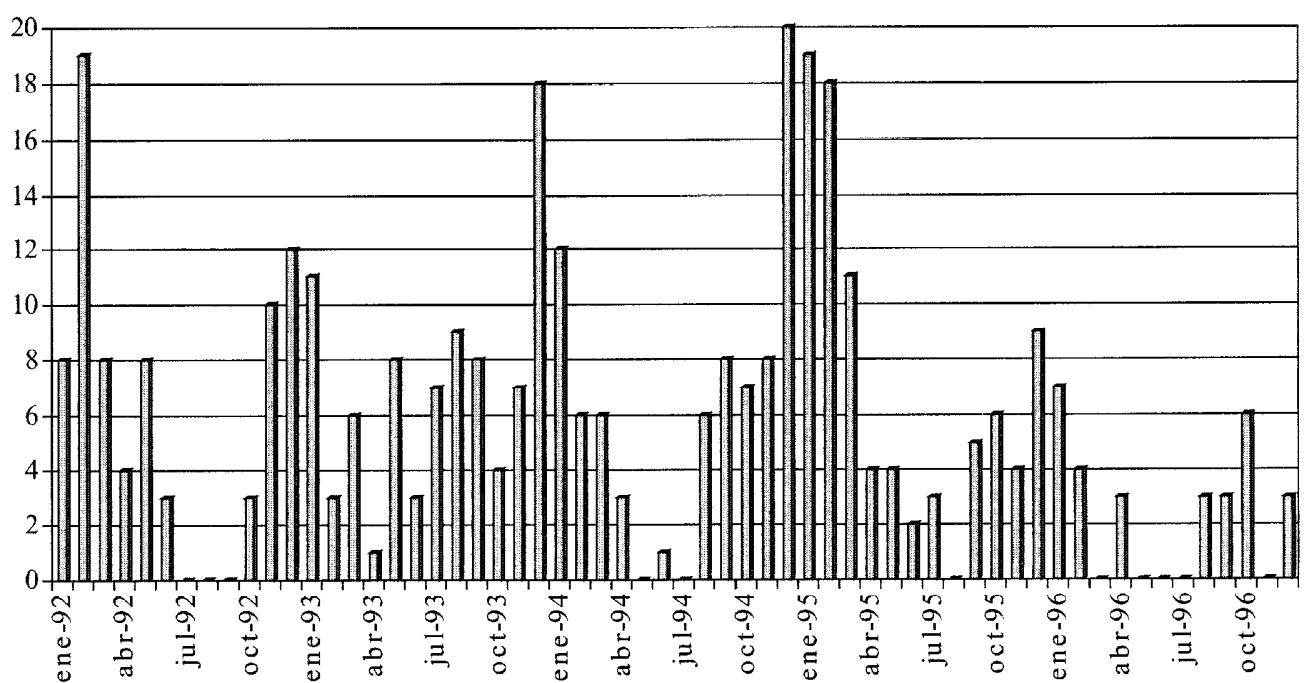


años (tabla 2). Las correlaciones entre los valores promedio de tempcratura, humedad relativa y los tipos de contaminantes atmosféricos, es baja en las tres estaciones captadoras. También es baja entre los índices de medición del dióxido de azufre y las partículas (tabla 3).

Al analizar todo el período desde 1992 a 1996, no se observaron asociaciones estadísticamente significativas entre las causas de defunción y los contaminantes atmosféricos estudiados (tabla 4). En todo el período, los modelos que mejor prueban la asociación entre contaminación y defunciones incluyen como máximo dos días después (retardo), excepto en la mortalidad por causa cardiovascular, que en su mayoría es el tercer día después.

En los resultados por semestres se evidenciaron asociaciones estadísticamente

Tabla 2

Estadísticos descriptivos de los indicadores diarios de defunciones, meteorología, gripe y contaminantes atmosféricos en la ciudad de Cartagena, 1992-1996

\begin{tabular}{|c|c|c|c|c|c|c|c|}
\hline & \multicolumn{5}{|c|}{ Periodo anual } & \multirow{2}{*}{$\begin{array}{l}\text { Semestre cálido } \\
\text { Media }\end{array}$} & \multirow{2}{*}{$\begin{array}{l}\text { Semestre frio } \\
\text { Media }\end{array}$} \\
\hline & Media & $\begin{array}{l}\text { Valor } \\
\text { minimo }\end{array}$ & $\begin{array}{l}\text { Valor } \\
\text { máximo }\end{array}$ & $\begin{array}{l}\text { Percentil } \\
\quad 10\end{array}$ & $\begin{array}{l}\text { Percentil } \\
\quad 90\end{array}$ & & \\
\hline Defunciones totales sin accidentes & 3.5 & 0 & 13 & 1 & 6 & 3,2 & 3,7 \\
\hline $\begin{array}{l}\text { Defunciones totales sin accidentes en } \\
\geq 70 \text { años de edad }\end{array}$ & 2.4 & 0 & 10 & 1 & 5 & 2,3 & 2,6 \\
\hline Defunciones cardiovasculares & 1.4 & 0 & 7 & 0 & 3 & 1,2 & 0,4 \\
\hline Defunciones respiratorias & 0.4 & 0 & 4 & 0 & 1 & 0,3 & 0,4 \\
\hline Temperatura media $\left({ }^{\circ} \mathrm{C}\right)$ & 19.1 & 7,2 & 30,8 & 12,4 & 26,5 & 23,2 & 14,9 \\
\hline Humedad relativa (\%) & 75.2 & 18,0 & 95,0 & 58,0 & 90,0 & 75,5 & 75,0 \\
\hline Gripe (casos incidentes) & 27.3 & 0 & 202 & 1 & 90 & 6,2 & 48,7 \\
\hline $\mathrm{SO} 2$ valor máximo horario $\left(\mu \mathrm{g} / \mathrm{m}^{3}\right)$ & 113.20 & 18,46 & 208,38 & 44,70 & 208,38 & 102,58 & 124,11 \\
\hline $\mathrm{SO} 2$ promedio $\left(\mu \mathrm{g} / \mathrm{m}^{3}\right)$ & 43,85 & 12,71 & 235,70 & 26,10 & 63,96 & 41,34 & 46,44 \\
\hline $\begin{array}{l}\text { Partículas valor máximo horario } \\
\left(\mu \mathrm{g} / \mathrm{m}^{3}\right)\end{array}$ & 178.42 & 27,56 & 689,00 & 72,51 & 331,63 & 157,68 & 199,70 \\
\hline Partículas promedio horario $\left(\mu \mathrm{g} / \mathrm{m}^{3}\right)$ & 55,89 & 12,23 & 226,96 & 31,45 & 85,63 & 52,36 & 59,52 \\
\hline
\end{tabular}

El semestre cálido comprende los meses de mayo a octubre.

F.l semestre frío comprende los meses de noviembre a abril.

Tabla 3

Correlaciones \& entre valores promedio diarios de los contaminantes atmosféricos, con la temperatura y la humedad relativa en las 3 estaciones captadoras, en la ciudad de Cartagena, 1992-1996

\begin{tabular}{|c|c|c|c|c|c|c|}
\hline & Temperatura & Humedad & $\begin{array}{l}\mathrm{SO}_{2} \text { valor } \\
\text { máximo }\end{array}$ & $\begin{array}{c}\mathrm{SO}_{2} \\
\text { promedio }\end{array}$ & $\begin{array}{l}\text { Partículas va- } \\
\text { lor máximo }\end{array}$ & $\begin{array}{l}\text { Particulas } \\
\text { promedio }\end{array}$ \\
\hline Temperatura $^{+}$ & 1,00 & $-0,11 *$ & $-0,23 *$ & $-0,18 *$ & $-0,16 *$ & $-0,05 *$ \\
\hline Humedad relativa ${ }^{\ddagger}$ & & 1,00 & $0,06 *$ & $-0,02$ & $-0,08 *$ & $0,07^{*}$ \\
\hline $\mathrm{SO}_{2}$ valor máximo & & & 1,00 & $0,81 *$ & $0,23 *$ & 0,25 \\
\hline $\mathrm{SO}_{2}$ promedio & & & & 1,00 & $0,17 *$ & $0,20 *$ \\
\hline Particulas valor máximo & & & & & 1,00 & $0,82 *$ \\
\hline Particulas promedio & & & & & & 1,00 \\
\hline
\end{tabular}

(†) Temperatura promedio de la máxima y mínima horaria diaria.

$\left(\begin{array}{c}+ \\ +\end{array}\right)$ Humedad relativa promedio de los registros a las 7,13 y 18 horas.

(\&) Coeficientes de correlación de Spearman.

(*) La correlación es estadisticamente significativa con $\mathrm{p} \leq 0,05$ (bilateral) 
Tabla 4

Asociación * entre contaminantes atmosféricos y causas de defunción en todo el período en la ciudad de Cartagena, 1992-1996

\begin{tabular}{|c|c|c|c|c|c|c|c|c|}
\hline & \multicolumn{2}{|r|}{$\begin{array}{l}\text { Defunciones totales } \\
\text { sin accidentes }\end{array}$} & \multicolumn{2}{|c|}{$\begin{array}{l}\text { Defunciones totales } \\
\text { sin accidentes en } \geq 70 \text { años }\end{array}$} & \multicolumn{4}{|c|}{$\begin{array}{c}\text { Definciones por enfermedades Defunciones por enfermedades } \\
\text { cardiocirculatorias } \\
\text { respiratorias }\end{array}$} \\
\hline & Ret & $R R(I C 95 \%)$ & $\operatorname{Ret}$ & $R R(I C 95 \%)$ & Ret & $R R(I C 95 \%)$ & Ret & $R R(I C 95 \%)$ \\
\hline $\mathrm{SO}_{2}$ valor máximo & 1 & 0,$9999 ; 0,9996$ a 1,0002 & 1 & 0,$9998 ; 0,9994$ a 1,000 & 3 & $1.000 ; 0,9997$ a 1.001 & 2 & 0,$9997 ; 0,998$ a 1.001 \\
\hline $\mathrm{SO}_{2}$ promedio & 2 & 0,$999 ; 0,998$ a 1,001 & 1 & 0,$9988 ; 0,9970$ a 1.000 & 2 & 0,$9984 ; 0,9962$ a 1.001 & 1 & 0,$9973 ; 0,9938$ a 1.002 \\
\hline Particulas valor máximo & 2 & 0,$9998 ; 0,9995$ a 1,0000 & 2 & 0,$9987 ; 0,9974$ a 1.000 & 3 & 1,$000 ; 1,000$ a 1,001 & 2 & 0,$9996 ; 0,9998$ a 1.000 \\
\hline Partículas promedio & 2 & 0,$9991 ; 0.9980$ a 1.000 & 2 & 0,$9987 ; 0,9974$ a 1.000 & 3 & $1.001 ; 0,9998$ a 1,003 & 0 & $1.002 ; 0,999$ a 1.006 \\
\hline
\end{tabular}

(*) Riesgo relativo e intervalo de confianza al $95 \%$ ajustado por estacionalidad, meteorología y gripe, para un incremento de nivel del contaminante atmosférico de $10 \mu \mathrm{g} / \mathrm{m}^{3}$.

Ret $=$ retardo.

significativas negativas entre el valor máximo de las partículas y las defunciones totales sin accidentes en personas de 70 y más años, y de signo positivo con el promedio de las partículas y las defunciones cardiovasculares del mes de mayo a octubre (tabla 5). Los modelos que mejor describen la relación contaminante/mortalidad llegan hasta el cuarto día para la manifestación del fallecimiento. La asociación positiva se da en el mismo día (retardo 0 en la tabla).

En el semestre frío, encontramos relación estadísticamente significativa y positiva en el valor máximo horario de las partículas y las muertes por entidades cardiocirculatorias y respiratorias, al tercer día del fallecimiento (tabla 5).

Cabría la posibilidad de hallar el RR para la diferencia entre los percentiles 90 y 10 , pero estos valores van a estar influenciados por el Plan de Intervención, por lo que sería poco usual días de intervención con mediciones altas de contaminación, dejando de ser un fenómeno aleatorio. Por ello se decidió la utilización del RR en unidades naturales de los contaminantes.

Tabla 5

Asociación * entre contaminantes químicos atmosféricos y causas de defunción en los semestres cálido y frío en la ciudad de Cartagena, 1992-1996

\begin{tabular}{|c|c|c|c|c|c|c|c|c|}
\hline & \multicolumn{2}{|c|}{$\begin{array}{c}\text { Defunciones totales sin } \\
\text { accidentes }\end{array}$} & \multicolumn{2}{|c|}{$\begin{array}{l}\text { Defunciones totales sin } \\
\text { accidentes en } \geq 70 \text { años }\end{array}$} & \multicolumn{4}{|c|}{$\begin{array}{l}\text { Defunciones por enferme-Defunciones por enferme- } \\
\text { dades cardiocirculatorias dades respiratorias }\end{array}$} \\
\hline & Ret & $R R(I C 95 \%)$ & Ret & $R R(I C 95 \%)$ & Ret & $R R(I C 95 \%)$ & Ret & $R R(I C 95 \%)$ \\
\hline \multicolumn{9}{|l|}{ Semestre cálido } \\
\hline $\mathrm{SO}_{2}$ valor máximo & 2 & $0,997(0,991-1,002)$ & 2 & $0,979(0,951-1,006)$ & 0 & $0,994(0,973-1,015)$ & 4 & $1,003(0,999-1,007)$ \\
\hline $\mathrm{SO}_{2}$ promedio & 4 & $1,006(0,999-1,012)$ & 2 & $0,972(0,941-1,005)$ & 2 & $0,979(0,955-1,004)$ & 2 & $0,996(0,991-1,001)$ \\
\hline Partículas valor máximo & 3 & $1,005(0,996-1,013)$ & $2 * *$ & $0,977(0,972-0,981)$ & 3 & $1,015(0,983-1,048)$ & 3 & $1,004(0,998-1,011)$ \\
\hline Partículas valor promedio & 1 & $0,993(0,975-1,011)$ & 1 & $0,945(0,864-1,033)$ & $\theta$ & $1,072(1.008-1,140)$ & 2 & $0,990(0,976-1,003)$ \\
\hline \multicolumn{9}{|l|}{ Semestre frio } \\
\hline $\mathrm{SO}_{2}$ valor máximo & 3 & $1,001(0,997-1,005)$ & 3 & $1,002(0,986-1,019)$ & 3 & $1,011(0,998-1,024)$ & 1 & $0,997(0,994-1,000)$ \\
\hline $\mathrm{SO}_{2}$ promedio & 1 & $0,997(0,992-1,002)$ & 1 & $0,986(0,965-1,007)$ & 3 & $1,014(0.998-1,029)$ & 3 & $1,003(0,999-1,006)$ \\
\hline Partículas valor máximo & 4 & $1,003(0,997-1,009)$ & 2 & $0,983(0,955-1,012)$ & $3 * *$ & $1,021(1,001-1,042)$ & $3^{* *}$ & $1,006(1,002-1,011)$ \\
\hline Particulas valor promedio & 5 & $0,995(0,981-1,009)$ & 4 & $0,976(0,922-1,033)$ & 3 & $1,021(0,981-1,064)$ & 0 & $1,006(0,996-1,015)$ \\
\hline
\end{tabular}

${ }^{*}$ ) Riesgo relativo e intervalo de confianza al $95 \%$ ajustado por estacionalidad, meteorologia y gripe, para un incremento de nivel del contaminante atmosférico de $10 \mu \mathrm{g} / \mathrm{m}^{3}$.

Semestre cálido incluye los meses de mayo a octubre y el frio de noviembre a abril.

Ret $=$ retardo en días. ${ }^{(* *)}$ El modelo de parametrización no es consistente. 
Al evaluar la fiabilidad de los modelos con riesgos relativos estadísticamente significativos, observamos que los modelos incluyen variables distintas, y que los coeficientes de regresión para el mismo contaminante presentan magnitudes y niveles de significación diferentes (tabla 6).

Los análisis por semestre elaborados con la totalidad de los datos del período que incluyen la interacción contaminante/semestre presentan una composición de variables y resultados distintos frente a los modelos que se construyen sólo con los datos del semestre. No obstante es necesa- rio, como parece lógico, que los resultados obtenidos sean interpretados con gran prudencia.

Podríamos plantear, como líneas de futuro, en primer lugar la de conocer el efecto de las intervenciones municipales en el control de la contaminación y, en segundo lugar, el aplicar los mismos modelos con la patología cardiovascular y la morbilidad respiratoria, y a su vez ésta con otra contaminación biológica como son los pólenes. Asimismo, será preciso caracterizar los efectos combinados de la contaminación atmosférica por $\mathrm{SO}_{2}$ y partículas.

Tabla 6

Fiabilidad de los modelos con asociaciones estadísticamente significativas entre contaminación atmosférica $y$ tipos de defunciones. EMECAM-Cartagena, 1992-96

\begin{tabular}{|c|c|c|c|c|}
\hline & \multirow{2}{*}{$\begin{array}{l}\text { Modelo con al contaminante } \\
\text { al inicio (modelo } 1) \\
\text { Coef. de regresión (E.E.) }\end{array}$} & \multirow{2}{*}{$\begin{array}{l}\text { Modelo con el contaminante } \\
\text { al final (modelo 2) } \\
\text { Coef. de regresión (E.E.) }\end{array}$} & \multicolumn{2}{|c|}{ Variables distintas entre los modelos } \\
\hline & & & $\begin{array}{l}\text { Modelo con el conta- } \\
\text { minante al inicio } \\
(\text { modelo } 1)\end{array}$ & $\begin{array}{l}\text { Modelo con el conta- } \\
\text { minante al final } \\
\text { (modelo 2) }\end{array}$ \\
\hline \multicolumn{5}{|l|}{ Semestre cálido } \\
\hline $\begin{array}{l}\text { Defunciones totales sin accidentes } \\
\text { en } \geq 70 \text { años y valor maximo en } \\
\text { Partículas* }\end{array}$ & $-0,4038 \mathrm{E}-03(0,251 \mathrm{E}-03)$ & $-0,5469 \mathrm{E}-03(0,257 \mathrm{E}-03)$ & $\begin{array}{l}\text { retardos } 2 \text { y } 7 \text { de tem- } \\
\text { peratura y } 2 \text { de tempe- }_{\text {ratura }^{2}}\end{array}$ & $\begin{array}{l}\text { retardo } 6 \text { de humedad } \\
\text { y dia de la semana e } \\
\text { intervención munici- } \\
\text { pal sobre la contami- } \\
\text { nación. }\end{array}$ \\
\hline $\begin{array}{l}\text { Defunciones cardiovasculares y va- } \\
\text { lor promedio en Particulas * }\end{array}$ & $0,1473 \mathrm{E}-02(0,165 \mathrm{E}-02)$ & $0,1742 \mathrm{E}-02(0,165 \mathrm{E}-02)$ & retardo 6 de humedad & eventos especiales \\
\hline \multicolumn{5}{|l|}{ Semestre frio } \\
\hline $\begin{array}{l}\text { Defunciones cardiovasculares y el } \\
\text { valor máximo en Partículas * }\end{array}$ & $0,6384 \mathrm{E}-03(0,233 \mathrm{E}-03)$ & $0,7671 \mathrm{E}-03(0,245 \mathrm{E}-03)$ & $\begin{array}{l}\text { tendencia }{ }^{2} \text {, retardo } 5 \\
\text { de gripe }\end{array}$ & $\begin{array}{l}\text { función sen/coseno2, } \\
\text { año de defunción }\end{array}$ \\
\hline $\begin{array}{l}\text { Defunciones respiratorias y el valor } \\
\text { máximo en Partículas * }\end{array}$ & $0,5524 \mathrm{E}-03(0,489 \mathrm{E}-03)$ & $0,4146 \mathrm{E}-03(0,489 \mathrm{E}-03)$ & retardo 10 de gripe & $\begin{array}{l}\text { retardo } 4 \text { de tempera- } \\
\text { tura, tendencia y ten- } \\
\text { dencia }^{2}\end{array}$ \\
\hline
\end{tabular}

Coef. $=$ coeficiente.

E.E. $=$ Error estándar.

(*) Modelo con distintas variables y distintas magnitudes y significación cstadistica de los coeficiente de regresión para el contaminante. 


\section{BIBLIOGRAFÍA}

1. Moreno Clavel, J. Repercusiones de la industrialización sobre nuestro ambiente. Cartagena: Escuela Universitaria de Ingeniería Industrial;1971.

2. Tormo MJ, Navarro C, Mortalidad general y por causas específicas e incidencia de cáncer en Cartagena y su comparación con la situación en otras áreas geográficas regionales. En: Contaminación atmosférica y salud en Cartagena. Cartagena: Consejeria de Sanidad; 1991. Monografias Sanitarias núm 10 .

3. Guillén Pérez JJ, Guillén Grima F, Medrano Tortosa J, García-Marcos L, Aguinaga Ontoso I, Niguez Carbonell JC. Afluencia inusual por asma y enfermedad pulmonar obstructiva crónica en urgencias hospitalarias y contaminación por SO2 en Cartagena. Rev Esp Salud Pública 1995;69:305-314.

4. Ballester Díez F, Sáez Zafra M, Alonso Fustel ME, Taracido Trunk M, Ordóñez Iriarte JM, Aguinaga Ontoso I et al. El proyecto EMECAM: Estudio multicéntrico español sobre la relación entre la contaminación atmosférica y la mortalidad. Antecedentes, participantes, objetivos, material y métodos. Rev Esp Salud Púb 1999: 73:165-175.

5. Pérez Hoyos $S$, Sáez Zafra M, Barceló Rado MA, Cambra K, Guillén Grima F, Bellido Blasco J, et al. Protocolo EMECAM: Análisis del efecto a corto plazo de la contaminación atmosférica sobre la mortalidad. Rev Esp Salud Púb 1999; 73:177-185.

6. Morcno Clavel J, Morcno J, Moreno Grau S, Jiménez Torres E. Operative plan for pollution control in Cartagena. Spain. En: Moreno Clavel J, Moreno Grau J. Proceedings of the workshop on best available technologies for pollution control.Luxemburgo: OPEUR

7. Ferrán Aranaz, M. SPSS para Windows. Programación y Análisis. Madrid: McGraw-Hill; 1996.

8. Abraira Santos V, Pérez de Vargas Luque A. Métodos Multivariantes en Bioestadística. Madrid: Centro de Estudios Ramón Areces SA; 1996. 